\title{
Studies on Morphological Characterization in Soft Rice (Oryza sativa L.) Genotypes
}

\author{
K. Pragnya ${ }^{1 *}$, K.V. Radha Krishna ${ }^{1}$, L.V. Subba Rao ${ }^{2}$ and K. Suneetha ${ }^{2}$ \\ ${ }^{1}$ Department of Genetics and Plant Breeding, College of Agriculture, PJTSAU, \\ Hyderabad-30, Telangana, India \\ ${ }^{2}$ Directorate of Rice Research, Rajendranagar, Hyderabad-30, Telangana, India
}

*Corresponding author

\section{A B S T R A C T}

Rice is the world's second most important cereal crop and staple food for more than 60 per cent of the global population. This paper focuses on morphological characterization using DUS discriptors for seventy five "speciality rices called soft rice genotypes" collected

\section{Keywords}

Characterization, DUS discriptors, Soft rice

Article Info

Accepted:

12 April 2018

Available Online:

10 May 2018 from different parts of north-east Assam. The lines were characterized for 62 agromorphological traits using DUS discriptors following UPOV guidelines (International Union for the Protection of New Varieties of Plants). The experiment was laid out in a randomized block design with three replications at Directorate of Rice Research Farm, ICRISAT Campus, Patancheru, Hyderabad, India. Among the 75 genotypes, some genotypes were monomorphic, some were bimorphic, some were trimorphic and the remaining genotypes exhibited large variation for some traits. On the whole significant variations were noticed for plant morphological characters, which is useful for each genotype to establish its diagnostic features. This high variability among the rice accessions studied, which presents great importance for breeding programs or for genetic studies on this species. This study will be useful for breeders, researchers and farmers to identify and choose the restoration and conservation of beneficial genes for crop improvement and also to seek protection under Protection of Plant Varieties and Farmer's Rights Act (PPV\&FR Act).

\section{Introduction}

The north-east region of India is a veritable natural gene bank representing wide spectrum of rice genetic resources. Among the different classes of rice available, glutinous / waxy rice is an important class, in the sense of being glue-like or sticky. The waxy rice of Assam has been classified in two groups viz., Bora (glutinous) and Chokuwa (Semi-glutinous) based on amylose content (Shaptadvipa and
Sarma, 2009). Bora rice of Assam has significance in social and religious ceremonies and forms a popular daily breakfast diet in rural Assam. Chokuwa (soft rice), is another class of rice used for instant preparations. This class of rice is not known in any other parts of the world. Its preparations are very popular in community feasts and festivals in Assam. "Soft rice (komal chawl)" are prepared from this class of rice by soaking the rice either in cold or hot water for a brief period of time and 
then consumed with sugar or molasses, milk or curd and even with salts and oils and pickles. These preparations seem to be useful for sailors, travelers, space researchers, mountaineers, defense personal etc. Thus this class of rice is metaphorically termed as "magical rice" as it becomes ready to use just by soaking with no fuel requirement and it has a great demand in the domestic as well as foreign market. The multiplicities of uses make the glutinous rice very popular among farmers. Inspite of the advent of modern high yielding rice varieties, these soft rices being mostly landraces are highly valuable and possess traits that are most preferred by farmers.

Until now, these cultivars have not grown outside the north-east, but the scientists at Central Rice Research Institute, Cuttak, have managed to develop a high yielding hybrid of a traditional soft rice variety called "Aghunibora". The field trials of the new hybrid were already positive, suggesting that it could be grown in different climates across India. Therefore we focused attention towards the improvement of both quality and yield potential of these soft rices to fetch high premium price in the market.

The global dependence on rice has led to the development of thousands of varieties with large genetic and morphological diversity. Therefore, the documentation of distinguishing characters is very essential to carry out the scientific study. Characterizing the genetic basis of variation in plants and linking it to the observable traits will provide an important framework for significantly increasing the efficiency of selections made in plant breeding programmes. Characterization of variety is useful to identify and avoid duplication.

Government of India has developed a 'Sui generis' system to provide a frame work for
Plant Variety Protection. In order to implement the sui generis system for plant variety protection for granting PBR to a breeder or farmer or institution, DUS (Distinctness, Uniformity and Stability) testing is compulsory. DUS testing involves the comparison between new candidate varieties with selected RCVs (Reference Collection Varieties) for a range of phenotypic characters, which are predominantly morphological traits. Initially, 62 agromorphological traits based to some extent on UPOV test guidelines were incorporated in the Indian National guidelines for the conduct of DUS tests in Rice.

Research in this direction on soft rice has been initiated and efforts are made by the Directorate of Rice Research for collection of these soft rice germplasm and land races. So far no work has been done on characterisation of these soft rice genotypes. The systematic characterisation studies in the material are meagre. Hence, keeping in view the importance of soft rices and scanty literature on these aspects, the present investigation was undertaken as a first attempt in the state of united Andhra Pradesh with the objective of Morphological characterization in soft rices of north-east region using DUS discriptors.

\section{Materials and Methods}

Present investigations were conducted at the Directorate of Rice Research Farm, ICRISAT Campus, Patancheru, Hyderabad, India. Seventy five (75) soft rice genotypes were characterized for 62 agro-morphological parameters. The experiment was laid out in a Randomized Block Design with three replications during the season. Thirty days old seedlings were transplanted at the rate of one seedling per hill in three rows of five metre length with plant to plant distance of $15 \mathrm{~cm}$ and row to row distance of $20 \mathrm{~cm}$. The standard cultivation practices prescribed for 
rice under irrigated conditions were followed precisely.

\section{Details of the experimental material}

The experimental material consist of seventy five soft rice genotypes obtained from the germplasm collections maintained at Directorate of Rice Research, Rajendranagar, Hyderabad and these genotypes were primarily collected from the north eastern region of Assam. The soft rice lines were given the notation as SR and the names of these soft rices were mentioned in table 1 .

\section{Recording of observations}

Five random plants were selected from central rows and the data was recorded in each replication on these five plants at different growth stages of the crop for characterization. The 62 agro-morphological characters studied were those given in DUS characterization of rice varieties (Subbarao et al., 2013) as per the national test guidelines.

\section{Results and Discussion}

In the present investigation, 75 soft rice genotypes were evaluated and characterized for morphological characters using DUS criteria as per the national guidelines for the conduct of DUS tests. Characterization was done for each genotype, to establish its diagnostic features.

The results of the observations recorded were presented in detail in table 2 and some important grain characters were depicted through figures.

\section{Coleoptile colour}

All the 75 genotypes under study had colourless coleoptiles and no variation was observed for this trait among these genotypes.

\section{Basal leaf: sheath colour}

Among the 75 genotypes evaluated 39 genotypes showed green basal leaf sheath colour, 14 genotypes showed light purple colour, 11 genotypes showed purple lines and the remaining 11 genotypes showed uniform purple colour. Similar work was reported by Bisne and Sarawgi (2008).

\section{Leaf: intensity of green colour}

Among the 75 genotypes evaluated, 27 genotypes showed light green leaf colour, 19 genotypes showed medium green colour and 29 genotypes had dark green colour. The intensity of green colour was found to be a useful trait to characterize the genotypes. Monika et al., (2007) also grouped nineteen varieties of rice based on intensity of green colour of leaf.

\section{Leaf: anthocyanin colouration}

Among the 75 genotypes evaluated 40 genotypes are devoid of anthocyanin coloration and 35 genotypes possessed leaf anthocyanin colouration. Similar type of work was carried out by Mageshwaran (2010) and Mathure et al., (2011).

\section{Leaf distribution of anthocyanin colouration}

Thirty five genotypes had anthocyanin colouration in the leaf. Of the 35 genotypes, anthocyanin is distributed only on tips for 13 genotypes and for 22 genotypes it is present on margins only.

Among the 75 genotypes studied there are no genotypes in the category where anthocyanin is neither distributed in blotches nor uniform distribution. Similar type of results was reported by Mageshwaran (2010) and Mathure et al., (2011). 


\section{Leaf sheath: anthocyanin colouration}

Among the 75 genotypes evaluated, anthocyanin colour in leaf sheath is absent for 41 genotypes and is present for 34 genotypes.

\section{Leaf sheath: intensity of anthocyanin colouration}

Among the 34 genotypes for which leaf sheath had anthocyanin colouration, two genotypes namely SR-34 and SR-35 had very weak intensity of anthocyanin colouration.

11 genotypes showed weak intensity, ten genotypes showed medium intensity, nine genotypes had strong intensity and the genotypes SR-6 and SR-49 had very strong leaf sheath intensity of anthocyanin colouration. Similar work was reported by Bisne and Sarawgi (2008) and Moukoumbi (2011).

\section{Leaf pubescence of blade surface}

Among the 75 genotypes studied, 37 genotypes showed medium pubesence, 19 genotypes showed strong genotypes and the remaining 19 genotypes weak pubesence. Evera (2003) used this trait to characterize twenty six rice cultivars.

\section{Leaf auricles}

Auricles are present in all 75 genotypes.

\section{Leaf: anthocyanin colouration of auricles}

Among the 75 genotypes, 35 genotypes had colourless auricles followed by 24 genotypes with light purple auricle and 16 genotypes with purple auricles.

Bisne and Sarawgi (2008) and Moukoumbi (2011) reported similar results on auricles characterization.

\section{Leaf collar}

Leaf collar is present in all the 75 genotypes. Similar results were obtained by Binse and Sarawgi (2008).

\section{Leaf: anthocyanin colouration of collar}

Among the 75 genotypes studied, 40 genotypes had anthocyanin in leaf collar and 35 genotypes had no anthocyanin colouration of collar. Similar work was reported by Bisne and Sarawgi (2008) and Moukoumbi (2011).

\section{Leaf ligule}

All the 75 genotypes had leaf ligule.

\section{Leaf: shape of ligule}

Of the 75 genotypes evaluated, all the genotypes showed split shaped ligule, and none of the genotypes showed truncate and acute shaped ligule. Evera (2003) used this trait to characterize twenty six paddy cultivars while Monika et al., (2007) and Bora et al., (2008a) used the same trait to characterize nineteen and eleven cultivars of rice respectively.

\section{Leaf: colour of ligule}

Among the 75 genotypes evaluated, 48 genotypes showed green ligule and 21 genotypes showed light purple ligule and six genotypes namely SR-10, SR-19, SR-33, SR51, SR-65 and SR-71 showed purple ligule.

\section{Leaf: length of the blade}

Wide variation was recorded for the length of the leaf blade. It ranged from $26.4 \mathrm{~cm}$ to 55.2 $\mathrm{cm}$. Of the 75 genotypes evaluated 56 genotypes are medium length, 10 genotypes are long length and 9 genotypes showed short length of the leaf blade. 


\section{Leaf: width of the blade}

All the 75 evaluated genotypes showed medium leaf width of the blade between 1-2 $\mathrm{cm}$. Similar variations in the length and width of the leaf were observed by Sharma et al., (2004). Rosta (1975) suggested that length and width of blade were quite useful traits in varietal identification.

Flag leaf: attitude of blade (early observation)

Fourty genotypes showed semi-erect flag leaf, followed by 19 genotypes showed erect flag leaf and 16 genotypes showed horizontal flag leaf and none of the genotypes showed deflexed flag leaf attitude of blade at early observation among the 75 genotypes evaluated (plate 4.10). Similar work was reported by Bisne and Sarawgi (2008), Moukoumbi (2011) and Sarawgi et al., (2012).

Flag leaf: attitude of blade (late observation)

Evaluation of 75 genotypes for flag leaf attitude of blade as late observation, 39 genotypes showed horizontal flag leaf, followed by 16 genotypes showed erect flag leaf, and 14 genotypes showed semi erect flag leaf and none of the genotypes showed deflexed flag leaf attitude of blade at late observation. Bora et al., (2008a) characterized eleven cultivars of rice using this character.

\section{Culm: attitude (for floating rice only)}

It is varied as procumbent and nonprocumbent. This trait is not applicable in the present investigation.

\section{Culm attitude}

Among the 75 genotypes, 53 genotypes had erect attitude of the culm, 14 were semi-erect followed by eight open type of culm. None of the genotypes had spreading culm attitude.

Time of heading $(50 \%$ of the plants with panicles)

Most of the genotypes i.e., 58 of the 75 were late duration (111-130 days) type and 17 genotypes took 91-110 days for flowering (medium duration).

Similar variation in time of heading was reported by Katsuta et al., (1996), Shah et al., (1999) and Santhy (1999) tested eight rice genotypes and found that days to flowering is a very important trait in classification of rice cultivars.

\section{Male sterility}

It is absent in all 75 genotypes.

\section{Lemma: anthocyanin colouration of keel}

Twenty two genotypes showed lack of anthocyanin colour, ten were weak, 21 genotypes had medium colouration, 19 genotypes with strong anthocyanin and three genotypes namely SR-6, SR-33 and SR-51 had very strong anthocyanin colouration of keel. Bisne and Sarawgi (2008), Moukoumbi (2011) and Sarawgi et al., (2012) reported similar results.

\section{Lemma: Anthocyanin colouration of area below apex}

About 27 of the 75 genotypes do not have colour below the apex, 14 genotypes are weakly coloured, 14 were medium, 16 were strong and four genotypes namely SR-2, SR-6, SR-8 and SR-51 had very strong anthocyanin colouration of area below apex. The results were in accordance with the work reported by Bisne and Sarawgi (2008), Moukoumbi (2011) and Sarawgi et al., (2012). 


\section{Lemma anthocyanin colouration of apex}

Among the 75 genotypes evaluated, 34 genotypes do not have anthocyanin colouration at the apex of lemma, one genotype (SR-29) had weak anthocyanin colouration, two genotypes (SR-35, SR-5) had medium colour, 16 genotypes had strong anthocyanin colouration and 22 genotypes with very strong anthocyanin colouration at the apex of lemmae. Monika et al., (2007) in nineteen rice varieties used this parameter for characterization.

\section{Spikelet: colour of stigma}

Of the 75 genotypes, 35 genotypes had white colour stigma, 23 genotypes with light green stigma, three genotypes namely SR-14, SR-27 and SR-75 were yellow, three genotypes (SR3, SR-35, SR-52) were light purple and 11 genotypes had purple stigma (plate 4.17). Chaudhury and Sahai (1993) found high variability for stigma colour while evaluating 1270 Cambodian rice genotype.

\section{Stem thickness}

All the genotypes had thin stem girth $(<0.40$ $\mathrm{cm})$ except SR-66 and SR-73 which had medium thickness.

\section{Stem length (excluding panicle)}

Thirty nine of the 75 genotypes had medium stem length followed by 22 genotypes with short stems, nine genotypes namely SR-21, SR-23, SR-26, SR-27, SR-28, SR-29, SR-30, SR-54 and SR-55 were very short and five genotypes (SR-1, SR-12, SR-40, SR-52, SR58) had long stem length. None of the genotype had very long stem length. A similar variation in plant height was reported by Rosta (1975), Anitalakshmi (2002), Nethra (2003) and Mageshwaran (2010) grouped rice genotypes based on this character.

\section{Stem: anthocyanin colouration of nodes}

Fourty four genotypes showed non pigmented anthocyanin colouration of nodes and 31 genotypes showed anthocyanin colouration of nodes.

\section{Stem: intensity of anthocyanin colouration of nodes}

Of the 31 genotypes which showed anthocyanin colouration of nodes, 16 genotypes showed strong intensity, 12 were medium and three genotypes (SR-20, SR-38, SR-44) had weak intensity of anthocyanin colouration of nodes.

Stem: anthocyanin colouration of inter nodes

Of the 75 genotypes evaluated, 38 genotypes had non pigmented inter nodes and 37 genotypes had anthocyanin colouration of inter nodes (plate 4.19). Similar work on nodes and inter nodes was reported by Bisne and Sarawgi (2008), Moukoumbi (2011) and Sarawgi et al., (2012).

\section{Panicle: length of main axis}

Eight genotypes (SR-4, SR-40, SR-41, SR-63, SR-65, SR-69, SR-70, SR-71) showed very long length of panicle, 37 genotypes had long length $(26-30 \mathrm{~cm}), 29$ genotypes had medium length $(21-25 \mathrm{~cm})$ and only one genotype (SR$73)$ showed short panicle length $(16-20 \mathrm{~cm})$ of main axis. Similar results were obtained by Sarma et al., (2004) where they characterized $142 a h u$ rice genotypes of Assam and found that eight genotypes showed more than $25 \mathrm{~cm}$ panicle length and the remaining genotypes recorded lesser panicle length.

Similar type of classification was reported by RohiniDevi (2000), Dhanaraj (2001) and Anitalakshmi (2002) in rice. 
Table.1 Details of soft rice genotypes used in the study

\begin{tabular}{|c|c|c|}
\hline S. No. & Soft rice line number & $\begin{array}{l}\text { Local name of the } \\
\text { genotype during collection }\end{array}$ \\
\hline 1 & SR-1 & Joha bora \\
\hline 2 & SR-2 & Ranga bora \\
\hline 3 & SR-3 & Sungal bora \\
\hline 4 & SR-4 & Noldong bora \\
\hline 5 & SR-5 & Tegori bora \\
\hline$\overline{6}$ & SR-6 & Bongari bora \\
\hline 7 & SR-7 & Kola ampaki bora \\
\hline 8 & SR-8 & Bora-1 \\
\hline 9 & SR-9 & Dadhora bora \\
\hline 10 & SR-10 & Chokura bora \\
\hline 11 & SR-11 & Sakoibhanu bora \\
\hline 12 & SR-12 & Kola bora \\
\hline 13 & SR-13 & Misiri chakua \\
\hline 14 & SR-14 & Boka chakua \\
\hline 15 & SR-15 & Ch-5 bora chakua \\
\hline 16 & SR-16 & Kagori chakura \\
\hline 17 & SR-17 & Kola boka chakura \\
\hline 18 & SR-18 & Haru chakua \\
\hline 19 & SR-19 & Boga chakua \\
\hline 20 & SR-20 & Lahi chakua \\
\hline 21 & SR-21 & Sam chakua \\
\hline 22 & SR-22 & Maju chakua \\
\hline 23 & SR-23 & Ham chakua \\
\hline 24 & SR-24 & Hampori chakua \\
\hline 25 & SR-25 & Malbhog \\
\hline 26 & SR-26 & Helochi \\
\hline 27 & SR-27 & Kalamdani \\
\hline 28 & SR-28 & Dadhora \\
\hline 29 & SR-29 & Aghoni bora \\
\hline 30 & SR-30 & Bhogali bora \\
\hline 31 & SR-31 & Abor bora \\
\hline 32 & SR-32 & Beji bora1 \\
\hline 33 & SR-33 & Begun bora \\
\hline 34 & SR-34 & Boga bora1 \\
\hline 35 & SR-35 & Boga bora 3 \\
\hline
\end{tabular}




\begin{tabular}{|c|c|c|}
\hline 36 & SR-36 & Bhat bora \\
\hline 37 & SR-37 & Bora 1 \\
\hline 38 & SR-38 & Bora 3 \\
\hline 39 & SR-39 & Bora 5 \\
\hline 40 & SR-40 & Botia bora \\
\hline 41 & SR-41 & Bor malbhog \\
\hline 42 & SR-42 & Chakkua bora 1 \\
\hline 43 & SR-43 & Chansep bora \\
\hline 44 & SR-44 & Chandra bora \\
\hline 45 & SR-45 & Danbori bora \\
\hline 46 & SR-46 & Fakkai bora \\
\hline 47 & SR-47 & Gela bora \\
\hline 48 & SR- 48 & Ghew bora 1 \\
\hline 49 & SR-49 & Garu chakua bora 2 \\
\hline 50 & SR-50 & Gomiri bora \\
\hline 51 & SR-51 & Naldang bora \\
\hline 52 & SR-52 & Helochi bora 1 \\
\hline 53 & SR-53 & Helochi bora 2 \\
\hline 54 & SR-54 & Aghoni \\
\hline 55 & SR-55 & Bhogali \\
\hline 56 & SR-56 & KMJ bora 56 \\
\hline 57 & SR-57 & KMJ bora 53 \\
\hline 58 & SR-58 & KMJ bora 51 \\
\hline 59 & SR-59 & KMJ bora 41 \\
\hline 60 & SR-60 & KMJ bora 36 \\
\hline 61 & SR-61 & KMJ bora49 \\
\hline 62 & SR-62 & KMJ bora 74 \\
\hline 63 & SR-63 & KMJ bora 5 \\
\hline 64 & SR-64 & KMJ bora 13 \\
\hline 65 & SR-65 & KMJ bora 21 \\
\hline 66 & SR-66 & KMJ bora 25 \\
\hline 67 & SR-67 & Boka chakua 1 \\
\hline 68 & SR-68 & Boka chakua 2 \\
\hline 69 & SR-69 & Kajoli chakua \\
\hline 70 & SR-70 & Kalamdani chakua \\
\hline 71 & SR-71 & Lahi chakua1 \\
\hline 72 & SR-72 & Maju chakua 1 \\
\hline 73 & SR-73 & Maju chakua 2 \\
\hline 74 & SR-74 & Misiri chakua \\
\hline 75 & SR-75 & Sam chakura \\
\hline
\end{tabular}


Table.2 Morphological characterization of soft rice genotypes

\begin{tabular}{|c|c|}
\hline 1. & $\begin{array}{l}\text { Coleoptile colour: } \\
\text { SR-1 to SR-75 have colourless coleoptiles. }\end{array}$ \\
\hline \multirow[t]{2}{*}{2.} & Basal leaf sheath colour \\
\hline & $\begin{array}{l}\text { Green- } 39 \text { genotypes- } \\
\text { SR-3, SR-7, SR-11, SR-12, SR-13, SR-15, SR-15, SR-16, SR-18, SR-21, SR-23, SR-24, SR-26, } \\
\text { SR-27, SR-28, SR-29, SR-30, SR-32, SR-34, SR-36, SR-37, SR-38, SR-39, SR-40, SR-42, SR- } \\
\text { 46, SR-48, SR-53, SR-54, SR-55, SR-60, SR-62, SR-64, SR-66, SR-67, SR-68, SR-69, SR-70 } \\
\text { and SR-74. } \\
\text { Light Purple - } 14 \text { genotypes } \\
\text { SR-1, SR-5, SR-17, SR-19, SR-20, SR-22, SR-25, SR-47, SR-50, SR-52, SR-65, SR-71, SR-72 } \\
\text { and SR-73. } \\
\text { Purple lines - } 11 \text { genotype } \\
\text { SR-2, SR-33, SR-35, SR-44, SR-45, SR-51, SR-56, SR-57, SR-58, SR-59 and SR-61. } \\
\text { Uniform purple- } 11 \text { genotypes } \\
\text { SRR-4, SR-6, SR-8, SR-9, SR-10, SR-31, SR-41, SR-43, SR-49, SR-63 and SR-75. }\end{array}$ \\
\hline \multirow[t]{2}{*}{3.} & Leaf: Intensity of green colour \\
\hline & $\begin{array}{l}\text { Light-27 genotypes } \\
\text { SR-1, SR-3, SR-5, SR-8, SR-12, SR-13, SR-17, SR-19, SR-24, SR-35, SR-36, SR-37, SR-39, } \\
\text { SR-42, SR-44, SR-45, SR-52, SR-57, SR-62, SR-63, SR-64, SR-65, SR-67, SR-69, SR-70, SR- } \\
71 \text { and SR-74. } \\
\text { Medium-19 genotypes } \\
\text { SR-2, SR-9, SR-10, SR-14, SR-18, SR-22, SR-25, SR-29, SR-32, SR-33, SR-40, SR-43, SR-46, } \\
\text { SR-47, SR-51, SR-53, SR-56, SR-61 and SR-73. } \\
\text { Dark-29 genotypes } \\
\text { SR-4, SR-6, SR-7, SR-11, SR-15, SR-16, SR-20, SR-21, SR-23, SR-26, SR-27, SR-28, SR-30, } \\
\text { SR-31, SR-34, SR-38, SR-41, SR-48, SR-49, SR-50, SR-54, SR-55, SR-58, SR-59, SR-60, SR- } \\
\text { 66, SR-68, SR-72 and SR-75. }\end{array}$ \\
\hline \multirow[t]{2}{*}{4.} & Leaf: anthocyanin colouration \\
\hline & $\begin{array}{l}\text { Absent-40 genotypes } \\
\text { SR-2, SR-3, SR-5, SR-11, SR-12, SR-13, SR-14, SR-15, SR-16, SR-20, SR-23, SR-26, SR-27, } \\
\text { SR-28, SR-29, SR-30, SR-31, SR-32, SR-34, SR-36, SR-37, SR-38, SR-39, SR-40, SR-42, SR- } \\
\text { 43, SR-45, SR-46, SR-51, SR-53, SR-54, SR-60, SR-62, SR-64, SR-66, SR-67, SR-68, SR-69, } \\
\text { SR-70 and SR-74. } \\
\text { Present-35 genotypes } \\
\text { SR-1, SR-4, SR-6, SR-7, SR-8, SR-9, SR-10, SR-17, SR-18, SR-19, SR-21, SR-22, SR-24, SR- } \\
\text { 25, SR-33, SR-35, SR-41, SR-44, SR-47, SR-48, SR-49, SR-49, SR-50, SR-52, SR-55, SR-56, } \\
\text { SR-57, SR-58, SR-59, SR-61, SR-63, SR-65, SR-71, SR-72, SR-73 and SR-75. }\end{array}$ \\
\hline \multirow[t]{2}{*}{5.} & Leaf: distribution of anthocyanin colou \\
\hline & $\begin{array}{l}\text { On tips only- } 13 \text { genotypes } \\
\text { SR-7, SR-17, SR-21, SR-24, SR-33, SR-44, SR-47, SR-48, SR-50, SR-55, SR-65, SR-71 and } \\
\text { SR-72 } \\
\text { On margins only- } 22 \text { genotypes } \\
\text { SR-1, SR-4, SR-6, SR-8, SR-9, SR-10, SR-18, SR-19, SR-22, SR-25, SR-35, SR-41, SR-49, SR- } \\
\text { 52, SR-56, SR-57, SR-58, SR-61, SR-63, SR-73, SR-75. } \\
\text { In bloches only - nil } \\
\text { Uniform - nil }\end{array}$ \\
\hline
\end{tabular}




\section{Leaf sheath anthocyanin colouration}

Absent - 41 genotypes

SR-1, SR-2, SR-7, SR-11, SR-12, SR-13, SR-14, SR-15, SR-16, SR-18, SR-20, SR-23, SR24, SR-26, SR-27, SR-28, SR-29, SR-30, SR-32, SR-36, SR-37, SR-39, SR-40, SR-42, SR-46, SR-47, SR-53, SR-54, SR-55, SR-57, SR-59, SR-60, SR-62, SR-64, SR-66, SR-67, SR-68, SR-69, SR-70, SR-71 and SR-74.

\section{Present- 34 genotypes}

SR-3, SR-4, SR-5, SR-6, SR-8, SR-9, SR-10, SR-17, SR-19, SR-21, SR-22, SR-25, SR-31, SR-33, SR-34, SR-35, SR-38, SR-41, SR-43, SR-44, SR-45, SR-48, SR-49, SR-50, SR-51, SR-52, SR-56, SR-58, SR-61, SR-63, SR-65, SR-72, SR-73 and SR-75.

7. Leaf sheath: intenssity of anthocyanin colouration

Very weak- two genotypes

SR-34 and SR-35.

\section{weak- 11 genotypes}

SR-31, SR-38, SR-45, SR-48, SR-52, SR-56, SR-58, SR-61, SR-72 and SR-73.

\section{Medium-10 genotypes}

SR-4, SR-19, SR-21, SR-22, SR-25, SR-41, SR-44, SR-51 and SR-65.

Strong- nine genotypes

SR-5, SR-8, SR-10, SR-17, SR-33, SR-43, SR-50, SR-63 and SR-75.

Very strong- two genotypes

SR-6 and SR- 49.

8. Leaf: pubesence of blade surface

\section{Absent- nil}

\section{Weak-19 genotypes}

SR-4, SR-8, SR-9, SR-13, SR-15, SR-29, SR-33, SR-36. SR-37, SR-42, SR-43, SR-44, SR46, SR-47, SR-48, SR-68, SR-69, SR-70 and SR-73.

Medium- 37 genotypes

SR-1, SR-5, SR-7, SR-10, SR-11, SR-12, SR-16, SR-18, SR-19, SR-20, SR-23, SR-25, SR26, SR-27, SR-30, SR-31, SR-35, SR-40, SR-41, SR-45, SR-50, SR-51, SR-52, SR-53, SR-55, SR-56, SR-57, SR-58, SR-60, SR-61, SR-62, SR-63, SR-64, SR-65, SR-66, SR-72 and SR-75.

Strong- 19 genotypes

SR-SR-2, SR-3, SR-6, SR-14, SR-17, SR-21, SR-22, SR-24, SR-28, SR-32, SR-34, SR-38, SR-39, SR-49, SR-54, SR-59, SR-67, SR-71 and SR-74.

Very strong- nil

9. Leaf auricles: present in all genotypes from SR-1 to SR-75.

10. Anthocyanin colouration of auricles

\section{Colourless- 35 genotypes}

SR-1, SR-7, SR-11, SR-12, SR-13, SR-14, SR-15, SR-18, SR-23, SR-24, SR-26, SR-27, SR29, SR-30, SR-32, SR-34, SR-35, SR-36, SR-37, SR-39, SR-42, SR-46, SR-49, SR-56, SR-57, SR-59, SR-60, SR-64, SR-66, SR-67, SR-68, SR-69, SR-70, SR-74 and SR-75.

\section{Light purple-24 genotypes}

SR-2, SR-3, SR-5, SR-8, SR-10, SR-16, SR-17, SR-19, SR-20, SR-22, SR-25, SR-31, SR-38, SR-41, SR-44, SR-45, SR-50, SR-53, SR-54, SR-55, SR-58, SR-65, SR-72 and SR-73.

Purple-16 genotypes

SR-4, SR-6, SR-9, SR-21, SR-33, SR-40, SR-43, SR-47, SR-48, SR-49, SR-51, SR-52, SR61, SR-62, SR-63 and SR-71. 
11. Leaf collar: present in all genotypes from SR-1 to SR-75.

12. Leaf: anthocyanin colouration of collar

Absent- 35 genotypes

SR-1, SR-7, SR-11, SR-12, SR-13, SR-14, SR-15, SR-16, SR-18, SR-23, SR-24, SR-26, SR-27, SR28, SR-29, SR-30, SR-32, SR-34, SR-36, SR-37, SR-38, SR-42, SR-45, SR-46, SR-53, SR-54, SR-SR56, SR-59, SR-60, SR-64, SR-66,SR-67, SR-68, SR-69 and SR-74.

Present- 40 genotypes

SR-2, SR-3, SR-4, SR-5, SR-6, SR-9, SR-10, SR-17, SR-19, SR-20, SR-21, SR-22, SR-25, SR-31, SR33, SR-35, SR-39, SR-SR-40, SR-41, SR-43, SR-44, SR-47, SR-48, SR-49, SR-50, SR-51, SR-52, SR55, SR-57, SR-58, SR-61, SR-62, SR-63, SR-65, SR-70, SR-71, SR-72, SR-73 and SR-75.

\section{3 \& Leaf ligule and shape of ligule}

14 All the genotypes from SR-1 to SR-75 have split shaped ligule.

15. Leaf colour of ligule

\section{Green- 48 genotypes}

SR-1, SR-2, SR-6, SR-7, SR-8, SR-9, SR-11, SR-12, SR-13, SR-15, SR-16, SR-18, SR-20, SR-21, SR22, SR-23, SR-24, SR-26, SR-28, SR-29, SR-30, SR-31, SR-36, SR-37, SR-38, SR-39, SR-40, SR-42, SR-43, SR-45, SR-46, SR-47, SR-49, SR-53, 54, SR-56, SR-59, SR-60, SR-61, SR-62, SR-63, SR-66, SR-67, SR-68, SR-70, SR-72, SR-73 and SR-75.

\section{Light purple- 21 genotypes}

SR-3, SR-4, SR-5, SR-14, SR-17, SR-25, SR-27, SR-32, SR-34, SR-35, SR-41, SR-44, SR-48, SR-50, SR-52, SR-55, SR-57, SR-58, SR-64, SR-69 and SR-74.

purple- 6genotypes

SR-10, SR-19, SR-33, SR-51, SR-65 and SR-71.

16. Leaf- length of the blade

Short- nine genotypes

SR-13, SR-28, SR-29, SR-30, SR-54, SR-55, SR-57, SR-65 and SR-66.

\section{Medium- 56 genotypes}

SR-1, SR-2, SR-3, SR-4, SR-5, SR-7, SR-8, SR-9, SR-10, SR-11, SR-12, SR-14, SR-15, SR-17, SR19, SR-21, SR-22, SR-23, SR-24, SR-25, SR-26, SR-31, SR-33, SR-34, SR-35, SR-36, SR-37, SR-39, SR-40, SR-41, SR-43, SR-44, SR-45, SR-46, SR-47, SR-48, SR-49, SR-51, SR-52, SR-56, SR-58, SR59, SR-60, SR-61, SR-62, SR-63, SR-64, SR-67, SR-68, SR-69, SR-70, SR-71, SR-72, SR-73, SR-74 and SR-75.

\section{Long- ten genotypes}

SR-6, SR-16, SR-18, SR-20, SR-27, SR-32, SR-38, SR-42, SR-50 and SR-53.

17. Leaf: width of the blade- SR-1 to SR-75 have medium leaf width.

18. Flag leaf attitude of the blade (early observation)

\section{Erect-19 genotypes}

SR-2, SR-5, SR-11, SR-15, SR-19, SR-20, SR-21, SR-23, SR-26, SR- 28, SR-29, SR-30, SR-36, SR44, SR-51, SR-55, SR-65, SR-68 and SR-74.

Semi erect-40 genotypes

SR-1, SR-3, SR-4, SR-6, SR-7, SR-8, SR-10, SR-14, SR-16, SR-18, SR-22,, SR-24 SR-27, SR-32, SR33, SR-34, SR-35, SR-37, SR-38, SR-41, SR-42, SR-43, SR-45, SR-47, SR-48, SR-49, SR-50, SR-52, SR-53, SR-54, SR-58, SR-59, SR-60, SR-62, SR-67, SR-68, SR-70, SR-71, SR-73 and SR-75.

Horizontal- 16 genotypes

SR-7, SR-9, SR-12, SR-13, SR-17, SR-25, SR-31, SR-39, SR-40, SR-46, SR-56, SR-61, SR-63, SR66, SR-69 and SR-72. 


\section{Flag leaf attitude of the blade (late observation)}

\section{Erect-16 genotypes}

SR-8, SR-20, SR-21, SR-23, SR-24, SR-26, SR-27, SR-28, SR-29, SR-30, SR-38, SR-43, SR-54, SR-55, SR-60 and SR-62.

Semi erect-14 genotypes

SR-5, SR-7, SR-11, SR-22, SR-32, SR-33, SR-34, SR-41, SR-45, SR-49, SR- 50, SR-51, SR-52 and SR-63.

\section{Horizontal- 39 genotypes}

SR-1, SR-2, SR-3, SR-4, SR-10, SR-12, SR-13, SR-14, SR- 15, SR-16, SR-18, SR19, SR-25, SR- 35, SR-36, SR-37, SR-39, SR-40, SR-42, SR-44, SR-46, SR-47, SR48, SR-53, SR-56, SR-57, SR-58, SR-59, SR-61, SR-65, SR-66, SR-67, SR-68, SR69, SR-71, SR-72, SR-73, SR-74 and SR-75.

Deflexed- six genotypes

SR-6, SR-9, SR-17, SR-31, SR-64 and SR-70.

20. Culm attitude (for floating rice only)- not applicable here.

\section{Culm attitude}

\section{Erect-53 genotypes}

SR-1, SR-3, SR-6, SR-7, SR-9, SR-10, SR-11, SR-12, SR-13, SR-14, SR-15, 16, SR17, SR-18, SR-19, SR-20, SR-21, SR-23, SR-24, SR-25, SR-27, SR-27, SR-28, SR29, SR-30, SR-31, SR-33, SR-36, SR-40, SR-41, SR-42, SR-44, SR-46, SR-48, SR49, SR-50, SR-51, SR-52, SR-54, SR-55, SR-56, SR-57, SR-58, SR-59, SR- 61, SR64, SR-66, SR-67, SR-68, SR-69, SR-71, SR-72, SR-73 and SR-75.

Semi erecct-14 genotypes

SR-4, SR-5, SR-8, SR-22, SR-26, SR-32, SR-38, SR-43, SR-45, SR-53, SR-60, SR63, SR-65 and SR-74.

\section{Open-eight genotypes}

SR-2, SR-34, SR-35, SR-37, SR-39, SR-47, SR-62 and SR-70.

Spreading- nil

\section{Time of heading ( $50 \%$ of plants with panicles)}

Very early- nil

Early- nil

\section{Medium -17 genotypes}

SR-1, SR-6, SR-9, SR-11, SR-14, SR-15, SR-16, SR-18, SR-23, SR-26, SR-31, SR39, SR-46, SR-49, SR-69, SR-71 and SR-72.

\section{Late- 58 genotypes}

SR-2, SR-3, SR-4, SR-5, SR-7, SR-8, SR-10, SR-12, SR-13, SR-17, SR-19, SR-20, SR-21, SR-22, SR-24, SR-25, SR-27, SR-28, SR-29, SR-30, SR-32, SR-33, SR-34, SR-35, SR-36, SR-37, SR-38, SR-40, SR-41, SR-42, SR-43, SR-44, SR-45, SR-47, SR-48, SR-50, SR-51, SR-52, SR-53, SR-54, SR-55, SR-56, SR-57, SR-58, SR-59, SR-60, SR-61, SR-62, SR-63, SR-64, SR-65, SR- 66, SR-67, SR-68, SR-70, SR-73, SR-74 and SR-75.

Very late- nil

23. Male sterility: Absent in all 75 genotypes 


\section{Lemma : Anthocyanin colouration of keel}

\section{Absent-22 genotypes}

SR-12, SR-13, SR-18, SR-20, SR-23, SR-24, SR-26, SR-27, SR-28, SR-32, SR-34, SR-36, SR-41, SR-42, SR-53, SR-54, SR-55, SR-61, SR-66, SR-68, SR-73 and SR-74.

\section{Weak-ten genotypes}

SR-5, SR-15, SR-22, SR-35, SR-39, SR-45, SR-46, SR-62, SR-69 and SR-70.

Medium-21 genotypes

SR-1, SR-3, SR-7, SR-11, SR-14, SR-21, SR-29, SR-37, SR-38, SR-40, SR-47, SR-48, SR50 ,

SR-56, SR-59, SR-60, SR-63, SR-64, SR-70, SR-72 and SR-75.

\section{Strong-19 genotypes}

SR-2, SR-4, SR-8, SR- 9, SR-10, SR-16, SR-17, SR-19, SR-25, SR-30, SR-31, SR-43, SR44, SR-49, SR-52, SR- 58, SR-65, SR-67 and SR-71.

Very strong- three genotypes

SR-6, SR-33 and SR-51.

\section{Lemma: anthocyanin colouration of area below apex}

Absent-27 genotypes

SR-3, SR-13, SR-15, SR-18, SR-20, SR-23, SR-26, SR-27, SR-28, SR-30, SR- 32, SR-34, SR-36, SR-37, SR-41, SR-42, SR-47, SR-53, SR-57, SR-58, SR-59, SR-61, SR-64, SR-66, SR-70 and SR-72.

\section{Weak-14 genotypes}

SR-7, SR-11, SR-12, SR- 29, SR-35, SR- 39, SR-40, SR-46, SR-48, SR-55, SR-60, SR-62, SR-73 and SR-74.

\section{Medium-14 genotypes}

SR-5, SR-9, SR-21, SR-22, SR-25, SR-28, SR-31, SR-38, SR-45, SR-50, SR-54, SR-56, SR-67 and SR-75.

\section{Strong-16 genotypes}

SR-1, SR-4, SR-10, SR-14, SR-16, SR-17, SR-19, SR-33, SR-43, SR-44, SR-49, SR-52, SR-63, SR-65, SR-69 and SR-71.

Very strong-4 genotypes

SR-2, SR-6, SR-8 and SR-51.

26. Lemma: anthocyanin colouration of apex

\section{Absent-34 genotypes}

SR-7, SR-11, SR-12, SR-13, SR-15, SR-21, SR- 23, SR-26, SR-27, SR-28, SR-30, SR-32, SR-34, SR-36, SR-37, SR-38, SR-42, SR-43, SR-46, SR-47, SR-48, SR-54, SR-57, SR-58, SR-59, SR-60, SR-61, SR-62, SR-64, SR-66, SR-67, SR-68, SR-70 and SR-74.

\section{Weak-1 genotype}

SR-29.

\section{Medium-two genotypes}

SR-5 and SR-35.

\section{Strong- 16 genotypes}

SR-3, SR-4, SR-9, SR-17, SR-18, SR-20, SR-22, SR-24, SR-39, SR-40, SR-41, SR-50, SR56, SR-72, SR-73 and SR-75.

Very strong- 22 genotypes

SR-1, SR-2, SR-6, SR-8, SR-10, SR-14, SR-16, SR-19, SR-25, SR-31, SR-33, SR-44, SR45, SR-49, SR-51, SR-52, SR-53, SR-55, SR-63, SR-65, SR-69 and SR-71. 


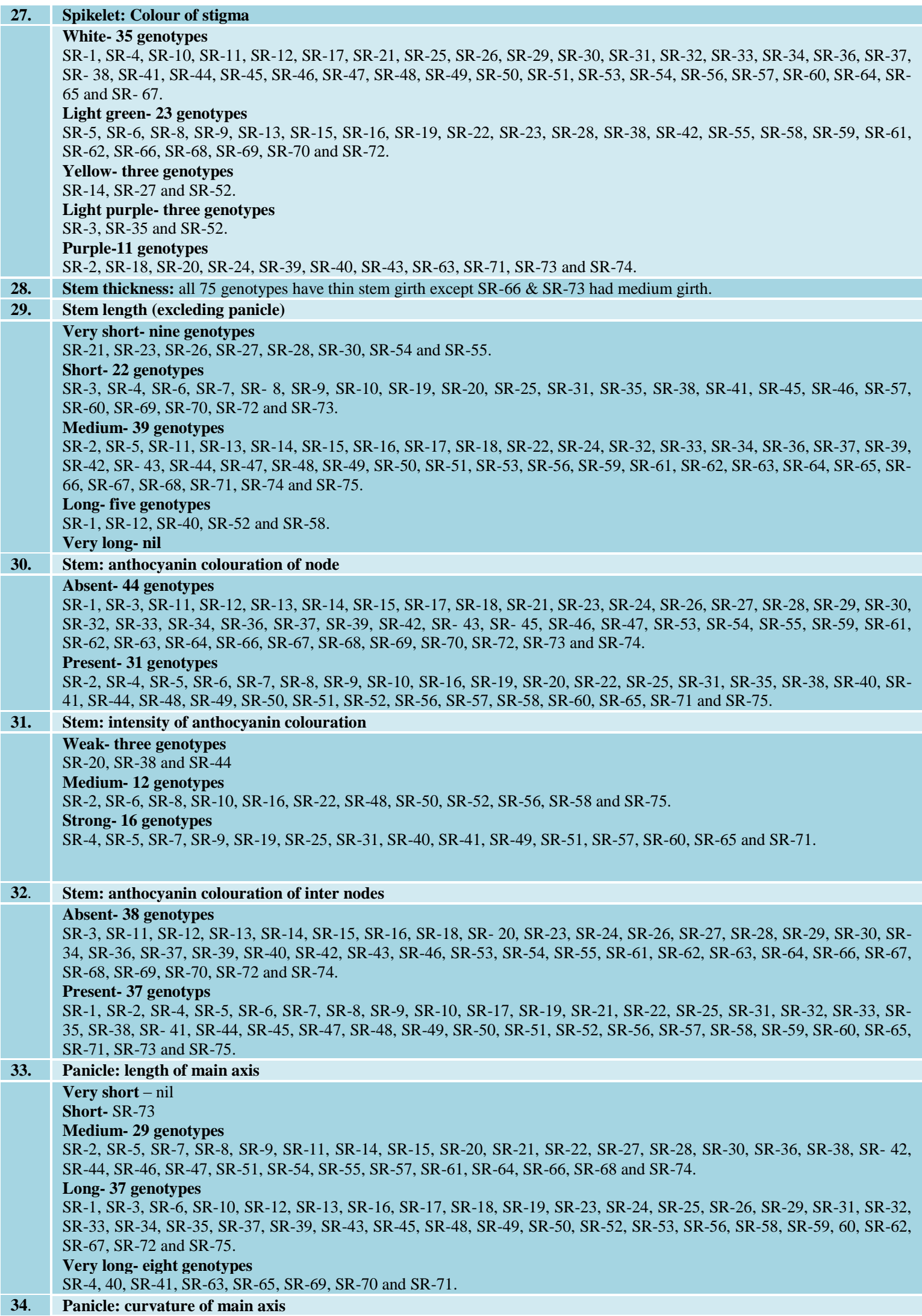




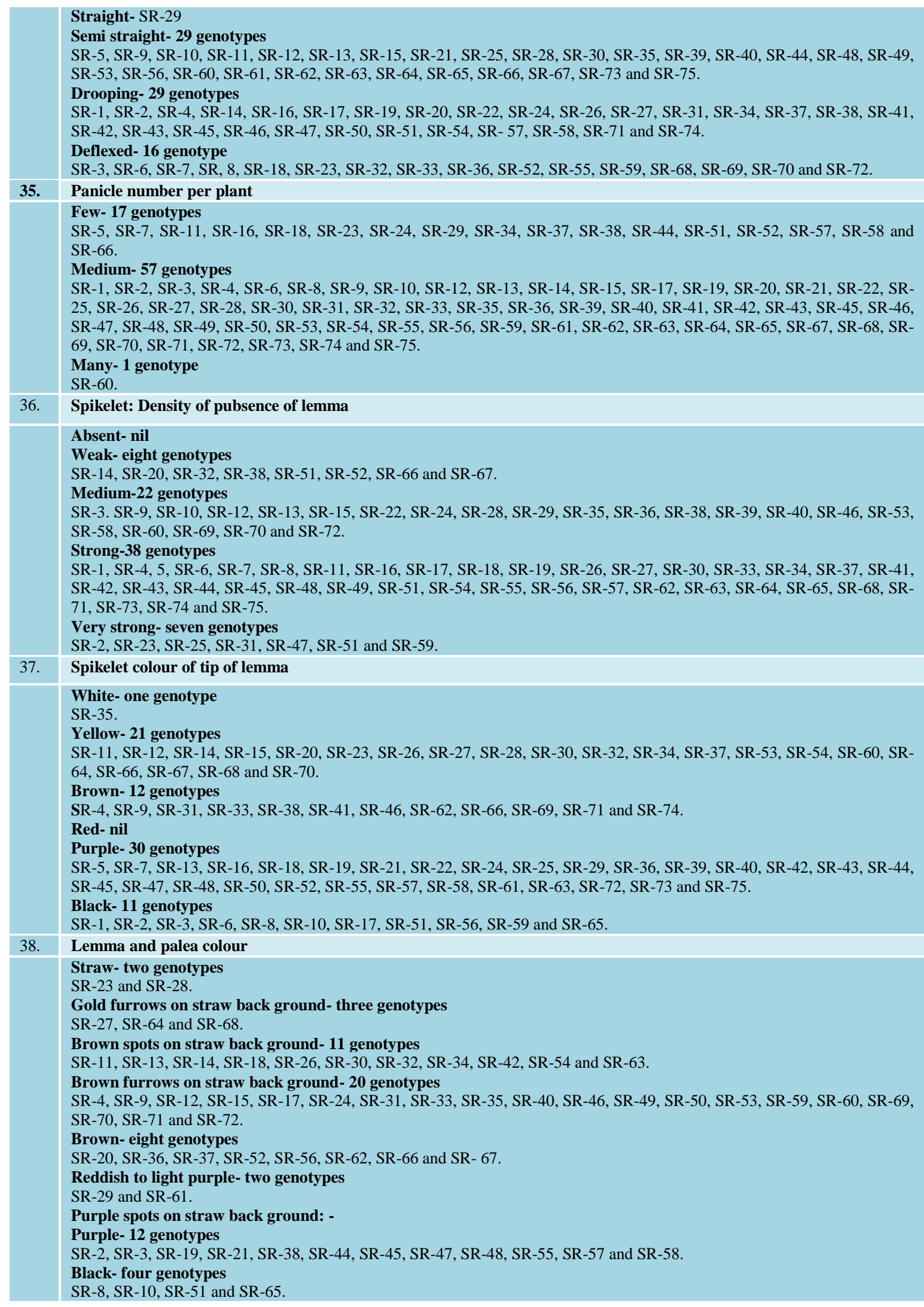


39. Panicle awns

Absent: 45 genotypes

SR-1, SR-5, SR-6, SR-7, SR-8, SR-9, SR-11, SR-13, SR-14, SR-15, SR-16, SR-17, SR-18, SR19, SR-20, SR-22, SR-28, SR-31, SR-33, SR-36, SR-37, SR-38, SR-39, SR-40, SR-42, SR-43, SR-46, SR-49, SR-50, SR-51, SR-52, SR-53, SR-56, SR-57 SR-59, SR-62, SR-64, SR-65, SR-66, SR-68, SR-69, SR-71, SR-73, SR-74 and SR-75.

Present- 30 genotypes

SR-2, SR-3, SR-4, SR-10, SR-12, SR-21, SR-23, SR-24, SR-25, SR-26, SR-27, SR-29, SR-30, SR-32, SR-34, SR-35, SR-41, SR-44, SR-45, SR-47, SR-48, SR-54, SR-55, SR-58, SR-60, SR61, SR-63, SR-67, SR-70 and SR-72.

40. Panicle: colour of awns (late observation)

Yellowish white- eight genotypes

SR-12, SR-27, SR-29, SR-30, SR-34, SR-54, SR-60 and SR-70.

Purple- 12 genotypes

SR-2, SR-4, SR-21, SR-25, SR-41, SR-45, SR-47, SR-48, SR-55, SR-58, SR-61 and SR-63.

Black- nil

41. Panicle: length of longest awns

Very short- three genotypes

SR-4, SR-25 and SR-63.

Short- nine genotypes

SR-2, SR-3, SR-12, SR-24, SR-29, SR-41, SR-47, SR-61 and SR-72.

Medium- 13 genotypes

SR-10, SR-21, SR-27, SR-30, SR-32, SR-34, SR-44, SR-45, SR-48, SR-54, SR-55, SR-67 and SR-70.

Long: four genotypes

SR-26, SR-35, SR-58 and SR-60.

Very long- one genotype

SR-23.

42. Panicle: distribution of awns

Tips only- 14 genotypes

SR-2, SR-3, SR-4, SR-12, SR-24, SR-29, SR-34, SR-41, SR-44, SR-47, SR-54, SR-61, SR-67 and SR-72.

Upper half only- nine genotypes

SR-10, SR-25, SR-30, SR-45, SR-48, SR-55, SR-60, SR-63 and SR-70.

Whole length- seven genotypes

SR-21, SR-23, SR26, SR-27, SR-32, SR-35 and SR-58.

43. Panicle: presence of secondary branching- present from SR-1 to SR-75.

44. Panicle- secondary branching

Weak- 14 genotypes

SR-5, SR-6, SR-10, SR-12, SR-19, SR-20, SR-22, SR-24, SR-25, SR-45, SR-50, SR-52, SR-59 and SR-69.

Strong- 54 genotypes

SR-1, SR-3, SR-4, SR-8, SR-9, SR-11, SR-13, SR-14, SR-15, SR-16, SR-17, SR-18, SR-21, SR23, SR-26, SR-28, SR-29, SR-30, SR-31, SR-32, SR-33, SR-34, SR-36, SR-37, SR-38, SR-39, 


\begin{tabular}{|c|c|}
\hline & $\begin{array}{l}\text { 56, SR-57, SR-58, SR-60, SR-61, SR-62, SR-65, SR-66, SR-67, SR-68, SR-70, SR-71, } \\
\text { SR-73, SR-74 and SR-75. } \\
\text { Clustering- seven genotypes } \\
\text { SR-2, SR-7, SR-27, SR-35, SR-43, SR-63 and SR-64. }\end{array}$ \\
\hline 45. & Panicle attitude of branches \\
\hline & $\begin{array}{l}\text { Erect- one genotype } \\
\text { SR-73. } \\
\text { Erect to semi erect- two genotypes } \\
\text { SR-37 and SR-38. } \\
\text { Semi erect- } 37 \text { genotypes } \\
\text { SR-2, SR-7, SR-11, SR-12, SR-13, SR-14, SR-15, SR-18, SR-21, SR-23, SR-26, SR-27, SR-28, } \\
\text { SR-29, SR-30, SR-32, SR-34, SR-35, SR-36, SR-39, SR-40, SR-42, SR-44, SR-46, SR-47, SR- } \\
\text { 48, SR-49, SR-51, SR-54, SR-56, SR-57, SR-58, SR-60, SR-61, SR-63, SR-64 and SR-72. } \\
\text { Semi erect to spreading- } 19 \text { genotypes } \\
\text { SR-1, SR-3, SR-4, SR-9, SR-17, SR-22, SR-24, SR-31, SR-43, SR-52, SR-53, SR-55, SR-59, } \\
\text { SR-62, SR-65, SR-66, SR-68, SR-70 and SR-75. } \\
\text { Spreading- 16 genotypes } \\
\text { SR-4, SR-5, SR-6, SR-8, SR-10, SR-16, SR-19, SR-20, SR-25, SR-33, SR-45, SR-50, SR-67, } \\
\text { SR-69, SR-71 and SR-74. }\end{array}$ \\
\hline 46. & Panicle exertion \\
\hline & $\begin{array}{l}\text { Partly exerted- } 14 \text { geenotypes } \\
\text { SR-7, SR-12, SR-13, SR-21, SR-23, SR-26, SR-27, SR-38, SR-41, SR-43, SR-53, SR-60, SR-66 } \\
\text { and SR-74. } \\
\text { Mostly exerted- } 23 \text { genotypes } \\
\text { SR-2, SR-3, SR-11, SR-18, SR-20, SR-29, SR-30, SR-34, SR-36, SR-37, SR-42, SR-45, SR-46, } \\
\text { SR-51, SR-52, SR-54, SR-56, SR-57, SR-63, SR-64, SR-65 and SR-70. } \\
\text { Well exerted- } 38 \text { genotypes } \\
\text { SR-1, SR-4, SR-5, SR-6, SR-8, SR-9, SR-10, SR-14, SR-15, SR-16, SR-17, SR-19, SR-22, SR- } \\
\text { 24, SR-25, SR-31, SR-32, SR-33, SR-35, SR-39, SR-40, SR-44, SR-47, SR-48, SR-49, SR-50, } \\
\text { SR-55, SR-58, SR-59, SR-61, SR-62, SR-67, SR-68, SR-69, SR-71, SR-72, SR-73 and SR-75. }\end{array}$ \\
\hline 47. & Time of maturity \\
\hline & $\begin{array}{l}\text { Very early- nil } \\
\text { Early- nil } \\
\text { Medium- } 15 \text { genotypes } \\
\text { SR-1, SR-6, SR-11, SR-14, SR-15, SR-18, SR-23, SR-26, SR-28, SR-46, SR-49, SR-69, SR-70, } \\
\text { SR-71 and SR-72. } \\
\text { Late- } 60 \text { genotypes } \\
\text { SR-2, SR-3, SR-4, SR-5, SR-7, SR-8, SR-9, SR-10, SR-12, SR-13, SR-16, SR-17, SR-19, SR-20, } \\
\text { SR-21, SR-22, SR-24, SR-25, SR-27, SR-29, SR-30, SR-31, SR-32, SR-33, SR-34, SR-35, SR- } \\
\text { 36, SR-37, SR-38, SR-39, SR-40, SR-41, SR-42, SR-43, SR-44, SR-45, SR-47, SR-48, SR-50, } \\
\text { SR-51, SR-52, SR-53, SR-54, SR-55, SR-56, SR-57, SR-58, SR-59, SR-60, SR-61, SR-62, SR- } \\
\text { 63, SR-64, SR-65, SR-66, SR-67, SR-68, SR-73, SR-74 and SR-75. } \\
\text { Very late- nil }\end{array}$ \\
\hline
\end{tabular}




\section{Leaf sensence}

\section{Early- 18 genotypes}

SR-8, SR-11, SR-14, SR-15, SR-20, SR-28, SR-35, SR-39, SR-40, SR-43, SR-45, SR-52, SR-54, SR-56, SR-59, SR-62, SR-70 and SR-72.

\section{Intermediate- 27 genotypes}

SR-3, SR-4, SR-10, SR-13, SR-16, SR-18, SR-22, SR-23, SR-25, SR-27, SR-29, SR30, SR-33, SR-34, SR-41, SR-44, SR-46, SR-49, SR-53, SR-57, SR-60, SR-61, SR63, SR-65, SR-68, SR-73 and SR-74.

\section{Late- 30 genotypes}

SR-1, SR-2, SR-5, SR-6, SR-7, SR-9, SR-12, SR-17, SR-19, SR-21, SR-24, SR-26, SR-31, SR-32, SR-36, SR-37, SR-38, SR-42, SR-47, SR-48, SR-50, SR-51, SR-55, SR-58, SR-64, SR-66, SR-67, SR-69, SR-71 and SR-75.

\section{Sterile lemma colour}

\section{Straw- 41 genotypes}

SR-2, SR-3, SR-5, SR-6, SR-11, SR-12, SR-13, SR-14, SR-15, SR-17, SR-18, SR-22, SR-23, SR-24, SR-26, SR-27, SR-28, SR-29, SR-30, SR-32, SR-34, SR-36, SR-37, SR-40, SR-42, SR-43, SR-49, SR-52, SR-53, SR-54, SR-55, SR-57, SR-60, SR-62, SR-63, SR-64, SR-66, SR-67, SR-68, SR-72 and SR-74.

\section{Gold- 10 genotypes}

SR-4, SR-8, SR-10, SR-16, SR-19, SR-31, SR-41, SR-46, SR-51 and SR-70.

\section{Red- nil}

\section{Purple-24 genotypes}

SR-1, SR-7, SR-9, SR-20, SR-21, SR-25, SR-33, SR-35, SR-38, SR-39, SR-44, SR45, SR-47, SR-48, SR-50, SR-56, SR-58, SR-59, SR-64, SR-65, SR-69, SR-71, SR73 and SR-75.

\section{Grain: weight of 1000 grain}

\section{Very low- one genotype}

SR-1.

\section{Low- two genotypes}

SR-59 and SR-60.

\section{Medium- 51 genotypes}

SR-3, SR-6, SR-7, SR-10, SR-11, SR-12, SR-13, SR-15, SR-16, SR-17, SR-19, SR-21, SR-22, SR-23, SR-25, SR-26, SR-27, SR-29, SR-30, SR-31, SR-32, SR-33, SR-37, SR-38, SR-39, SR-41, SR-42, SR-44, SR-45, SR-46, SR-47, SR-48, SR-49, SR-50, SR-51, SR-53, SR-54, SR-55, SR-56, SR-57, SR-61, SR-62, SR-64, SR-65, SR-66, SR-67, SR-68, SR-69, SR-73, SR-74 and SR-75.

\section{High- 17 genotypes}

SR-2, SR-4, SR-5, SR-8, SR-9, SR-14, SR-28, SR-34, SR-35, SR-36, SR-40, SR43, SR-52, SR-63, SR-70, SR-71 and SR-72.

\section{Very high- four genotypes}

SR-18, SR-20, SR-24 and SR-58. 


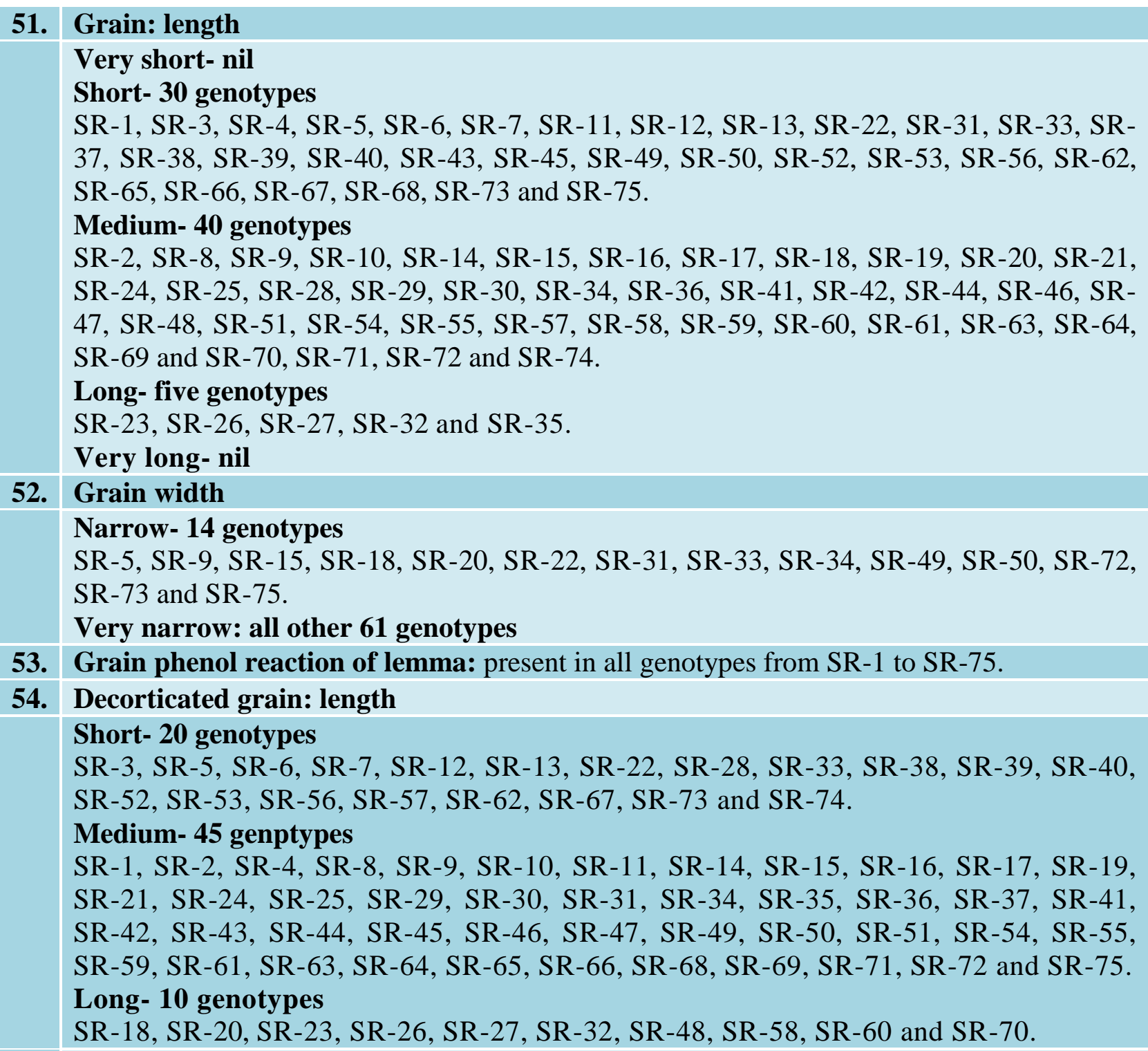

55. Decorticated grain: width- all genotypes have narrow decorticated grain width.

56. Decorticated grain: shape

\section{Short slender- 47 genotypes}

SR-1, SR-3, SR-4, SR-5, SR-6, SR-7, SR-8, SR-9, SR-10, SR-11, SR-12, SR-13, SR14, SR-15, SR-17, SR-19, SR-21, SR-24, SR-25, SR-29, SR-31, SR-33, SR-34, SR-36, SR-37, SR-38, SR-39, SR-40, SR-41, SR-42, SR-45, SR-46, SR-49, SR-50, SR-51, SR-52, SR-53, SR-56, SR-65, SR-66, SR-67, SR-68, SR-71, SR-72, SR-73, SR-74 and SR-75.

\section{Medium slender- 5 genotypes}

SR-22, SR-28, SR-30, SR-61 and SR-62.

\section{Long slender- 19 genotypes}

SR-2, SR-16, SR-18, SR-32, SR-35, SR-43, SR-44, SR-47, SR-48, SR-54, SR-55, SR57, SR-58, SR-59, SR-60, SR-63, SR-64, SR-69 and SR-70.

Basmati type- four genotypes 
SR-20, SR-23, SR-26 and SR-27.

57. Decorticated grain colour

Red- 13 genotypes

SR-12，SR-13，SR-16，SR-39，SR-40，SR-45，SR-53，SR-56，SR-57，SR-60, SR-65, SR-67, and SR-69.

White- other 62 genotypes

58. Endosperm: content of amylose- present in all genotypes from SR-1 to SR-75.

59. Endosperm content of amylose

Very low- 38 genotypes

SR-2, SR-3, SR-7, SR-8, SR-9, SR-10, SR-11, SR-12, SR-13, SR-21, SR-29, SR-30, SR35, SR-36, SR-37, SR-38, SR-40, SR-42, SR-43, SR-44, SR-46, SR-47, SR-48, SR-52, SR-53, SR-54, SR-55, SR-57, SR-58, SR-59, SR-60, SR-61, SR-63, SR-64, SR-65, SR67, SR-70 and SR-74.

\section{Low- 27 genotypes}

SR-1, SR-4, SR-5, SR-14, SR-15, SR-17, SR-18, SR-19, SR-20, SR-22, SR-23, SR-24, SR-25, SR-31, SR-32, SR-34, SR-39, SR-41, SR-45, SR-49, SR-50, SR-56, SR-68, SR71, SR-72, SR-73 and SR-75.

Itermediate- nine genotypes

SR-5, SR-16, SR-27, SR-28, SR-33, SR-51, SR-62, SR-66 and SR-69.

High- one genotype

SR-26.

Very high- nil

60. Varieties with endosperm of amylose absent: expression of white core

61. Gelatinization temperature through alkali spreading value

\section{Low- 36 genotypes}

SR-4, SR-5, SR-6, SR-7, SR-8, SR-11, SR-14, SR-17, SR-21, SR-22, SR-23, SR-27, SR28, SR-29, SR-30, SR-32, SR-33, SR-34, SR-36, SR-37, SR-38, SR-41, SR-42, SR-45, SR-46, SR-47, SR-48, SR-49, SR-50, SR-54, SR-55, SR-57, SR-58, SR-59, SR-60 and SR-75.

Medium- 36 genotypes

SR-1, SR-2, SR-3, SR-9, SR-10, SR-12, SR-13, SR-15, SR-16, SR-19, SR-20, SR-24, SR-26, SR-31, SR-35, SR-39, SR-40, SR-43, SR-44, SR-51, SR-52, SR-56, SR-61, SR62, SR-63, SR-64, SR-65, SR-66, SR-67, SR-68, SR-69, SR-70, SR-71, SR-72, SR-73 and SR-74.

High medium- three genotypes

SR-18, SR-25 and SR-53.

62. Decorticated grain aroma

Present- two genotypes

SR-23 and SR-26

Abent- all other genotypes 


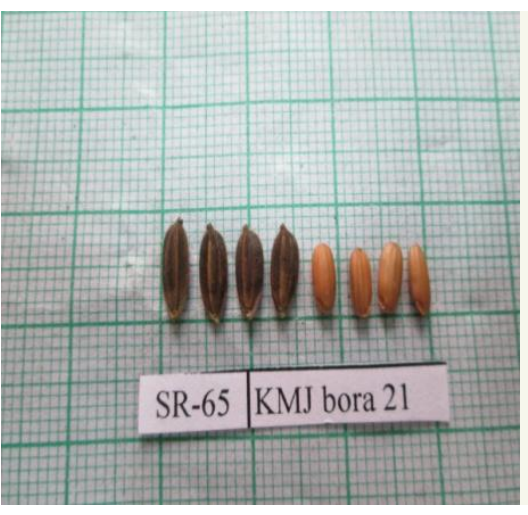

a. Grain and decorticated grain length- medium

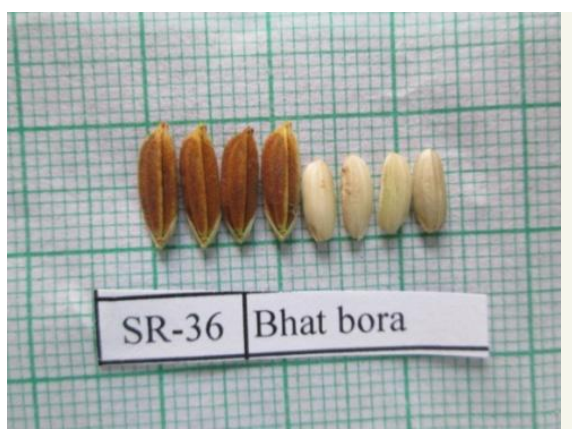

c. Grain and decorticated grain width- medium

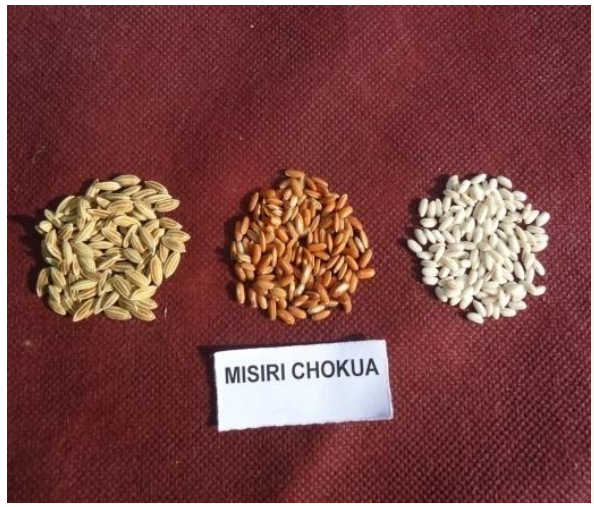

e. Decorticated grain colour- red

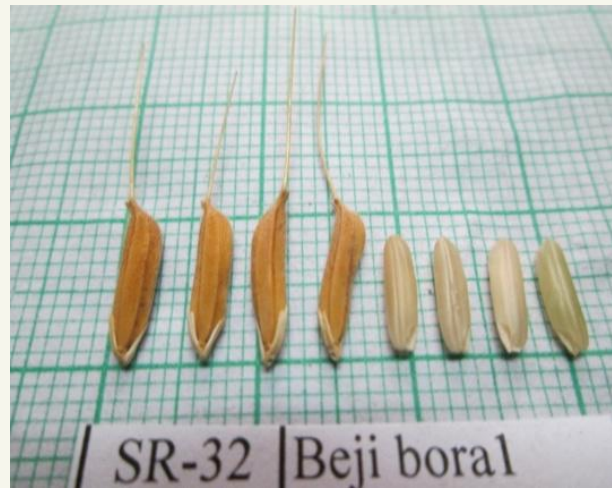

b. Grain and decorticated grain length- long

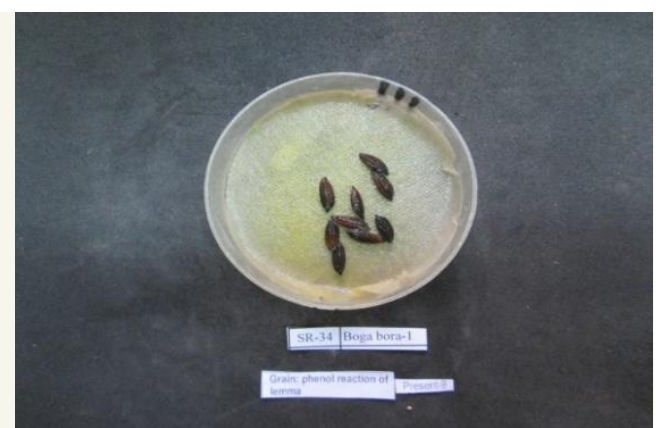

d. Grain: phenol reaction of lemma

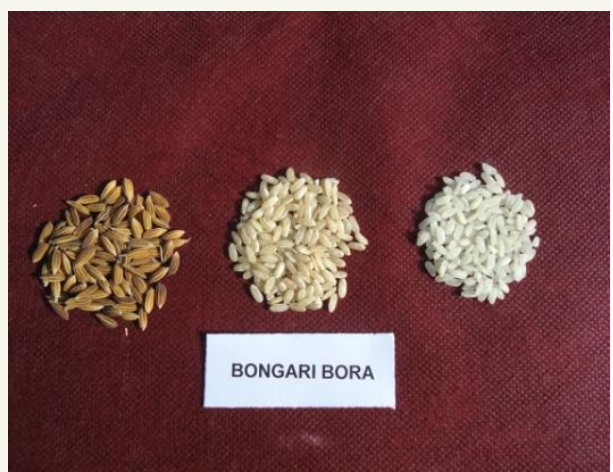

f. Decorticated grain colour- white

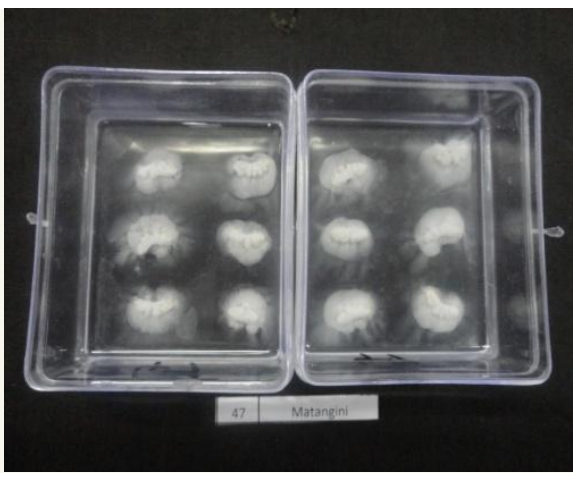

g. Gelatinization temparature through alkali spreading value- medium 


\section{Panicle: curvature of main axis}

Among the 75 genotypes evaluated, one straight panicle, 29 genotypes with semi straight panicles followed by 29 genotypes with drooping panicles, 16 genotypes had deflexed panicle curvature of main axis and one genotype (SR-29) had straight panicle (plate 4.21).

Similar work was reported by Bisne and Sarawgi (2008), Moukoumbi (2011) and Sarawgi et al., (2012).

\section{Panicle: number per plant}

Most of the genotypes, 57 of the 75 had medium number of panicles per plant (11-20), 17 genotypes had few panicles and only one genotype, SR-60 with many panicles $(>20)$, per plant. Monika et al., (2007) distinguished nineteen and eleven number of rice cultivars respectively based on panicle number per plant.

\section{Spikelet: density of pubescence of lemma}

Thirty eight genotypes showed strong pubescence of lemma, Medium pubescence of lemma was shown by 22 genotypes, followed by eight genotypes (SR-14, SR-20, SR-32, SR-38, SR-51, SR-52, SR-66 and SR-67) with weak pubescence of lemma and seven (SR-2, SR-23, SR-25, SR-31, SR-47, SR-51 and SR$59)$ with very strong pubescence, and none of the genotypes were non pubesent. Bora et al., (2008a) suggested that density of pubescence of lemma was a very important character for varietal characterization of rice.

\section{Spikelet: colour of tip of lemma}

About 30 genotypes showed purple lemma tip followed by 21 yellow, 12 had brown tip, 11 showed black lemma tip and one with white lemma tip.

\section{Lemma and palea colour}

Out of the 75 genotypes, 20 showed brown furrows on straw back ground, 13 had purple furrows on straw back ground, 12 were purple, 11 had brown spots on straw back ground, eight genotypes had brown lemma and palea colour, four (SR-8, SR-10, SR-51 and SR-65) had black colour, three (SR-27, SR-64 and SR-68) with gold furrows on straw back ground, two (SR-29 and SR-61) had reddish to light purple and two (SR-23 and SR-28) showed straw colour lemma and palea. Similar work was reported by Bisne and Sarawgi (2008), Moukoumbi (2011) and Sarawgi et al., (2012).

\section{Panicle awns}

Of the 75 genotypes, 45 are awnless and 30 are awned.

\section{Panicle: colour of awns (late observation)}

Among the 30 genotypes with awns, 12 were purple followed by eight yellowish white, four (SR-23, SR-26, SR-32 and SR-67) were yellowish brown, three (SR-3, SR-10 and SR72) brown and three genotypes namely SR24, SR-35 and SR-44 had red colour of awns.

\section{Panicle: length of longest awns}

Thirteen genotypes had medium length awns, nine genotypes showed short awns, three (SR4, SR-25 and SR-63) were very short, four (SR-26, SR-35, SR-58 and SR-60) long and only one genotype SR-23 had very long length awns.

\section{Panicle: distribution of awns}

Fourteen genotypes had awns on tips only, nine had awns only on upper half and seven genotypes namely SR-21, SR-23, SR26, SR27, SR-32, SR-35 and SR-58 showed awns on 
whole length of the panicle (plate 4.25). Similar type of classification was reported by Rohini Devi (2000), Dhanaraj (2001) and Anitalakshmi (2002) in rice.

\section{Panicle: presence of secondary branching}

All the 75 genotypes had secondary branching of the panicles.

\section{Panicle: secondary branching}

About 54 genotypes had strong secondary branching, 14 genotypes with weak secondary branching and seven genotypes namely SR-2, SR-7, SR-27, SR-35, SR-43, SR-63 and SR64 showed clustered secondary branching of the panicle (plate 4.27 and 4.28). Similar work was reported by Bisne and Sarawgi (2008), Moukoumbi (2011) and Sarawgi et al., (2012).

\section{Panicle: attitude of branches}

About 37 genotypes showed semi erect branches, 19 had semi erect to spreading type, 16 were spreading, ten genotypes namely SR37 and SR-38 were spreading and only one genotype SR-73 had erect attitude of branches.

\section{Panicle exsertion}

About 38 genotypes with well exerted panicle followed by 23 mostly exerted panicles and 14 genotypes with partly exerted panicles. The extent of panicle exsertion is important character in achieving successful pollination. This character is not only useful for characterization of genotypes per se but also to assess their suitability in hybrid development.

\section{Time of maturity}

Most of the genotypes (60 of the 75) took long time for maturity (140-160 days) and the remaining 15 genotypes took medium time for maturity.

\section{Leaf sensence}

Out of the 75 genotypes evaluated 30 genotypes recorded late leaf sensence, 27 genotypes with intermediate leaf sensence and 18 genotypes showed early leaf sensence.

\section{Sterile lemma colour}

Forty one genotypes with straw sterile lemma colour, followed by 24 genotypes with purple colour and 10 genotypes had golden colour sterile lemma and none of the genotypes showed red coloured sterile lemma.

\section{Grain: weight of 1000 fully developed grains}

The grain weight of 1000 fully developed grains ranged from $14.5 \mathrm{~g}$ to $33.16 \mathrm{~g}$ with a mean of $24.40 \mathrm{~g}$. Highest number of the genotypes (51) showed medium grain weight (21-25 g), followed by 17 genotypes with high grain weight (26-30 g), four genotypes (SR-18, SR-20, SR-24 and SR-58) showed very high (> $31 \mathrm{~g})$ grain weight, two genotypes namely SR-59 and SR-60 with low grain weight (15-20 g), only one (SR-1) genotype with very low weight $(<15 \mathrm{~g})$.

Thousand grain weight has been used for characterizing rice varieties by several workers, Bose and Pradhan (2005), Joshi et al., (2007).

\section{Grain length}

Forty genotypes with medium grain length (8.6-10.5), 30 were short length grain and five genotypes namely SR-23, SR-26, SR-27, SR32 and SR-35 had long grain length.

None of the genotypes showed very short or very long grain length (plate $a$ and $b$ ). 


\section{Grain width}

Of the 75 genotypes evaluated, 61 genotypes showed very narrow grain $(<2 \mathrm{~mm})$ and 14 genotypes had narrow grain width (plate c.). Similar variations in grain length and width were observed by Gupta (1990) and Sharma et al., (1990).

\section{Grain phenol reaction of lemma}

All the 75 genotypes showed positive response to phenol reaction of lemma (plate d.).

\section{Decorticated grain length}

Of the 75 genotypes studied, 45 genotypes have medium decorticated grain length (5.51$6.50 \mathrm{~mm}$ ) followed by 20 were short length $(<5.5 \mathrm{~mm})$ and 10 genotypes were long (6.51$7.5 \mathrm{~mm})$. None of them have extra-long length (plate (a) and (b)).

\section{Decorticated grain width}

All the 75 evaluated genotypes showed narrow decorticated grain width $(<2 \mathrm{~mm})$ (plate c.).

\section{Decorticated grain shape}

Forty seven genotypes have short slender shape followed by 19 with long slender, five were medium slender and four genotypes were in basmati grain shape.

\section{Decorticated grain colour}

Sixty two of the 75 genotypes had white decorticated grain colour and the remaining 13 genotypes had red colour (plate e \& f). None of the genotypes were found with any other colour. Decorticated Seed colour which is a heritable character has been used by several workers to distinguish crop genotypes, particularly in rice Nehra (2003), in cotton Reddy (2005) and in sesame Suhasini (2006).

Endosperm: presence of amylose

All the 75 evaluated genotypes had amylose in the endosperm.

\section{Endosperm content of amylose}

About 38 genotypes had very low amylose (39\%), 27 genotypes with low amylose content, nine genotypes were intermediate and only one genotype (SR-26) with high amylose in the endosperm. None of the genotypes had very high endosperm content of amylose.

Varieties with endosperm of amylose absent: expression of white core

This is not appicable in the present investigation.

\section{Gelatinization temperature through alkali} spreading values

Of the 75 genotypes 36 had low gelatinization temperature, 36 had medium gelatinization temperature (plate g.) and three genotypes (SR-18, SR-25 and SR-53) showed high gelatinization temperature.

\section{Decorticad grain aroma}

Only two genotypes, SR-23 and SR-26 were aromatic and all the other genotypes were devoid of aroma. Das and Ghosh (2010) studied four hundred thirty one traditional rice cultivars from genotype collection of Rice Research Station, Chinsurah, characterization had been done on thirty one traits.

The present investigation entitled "Studies on Morphological characterization in soft rice (Oryza sativa L.) genotypes" it can be summerised that morphological features play 
a major role in cultivar identification because they provide easy identification through quick visualization. In present investigation among the 62 agro-morphological characters, most of the characters showed variations in different accessions except colourless coleoptile colour, presence of leaf auricles, presence of leaf colour, presence of leaf ligule, shape of the ligule, width of the leaf blade, absence of male sterility, presence of secondary branching, phenol reaction of lemma and decorticated grain width and endosperm content of amylase, which were found to be monomorphic. In addition, leaf distribution of anthocyanin colouration, time of heading, stem thickness, time of maturity, grain width, decorticated grain length and decorticated grain colour were reported to be bimorphic. Basal leaf sheath colour, leaf intensity of green colour, leaf pubesence of blade surface, anthocyanin colouration of auricles, colour of the ligule, length of the leaf blade, flag leaf attitude of blade (early observation), culm attitude, intensity of anthocyanin colouration of nodes, panicle curvature of main axis, panicle number per plant, distribution of awns on panicle, secondary branching of the panicle, panicle exsertion, leaf senescence, sterile lemma colour, grain length and gelatinization temperature through alkali spreading value, which were found to be trimorphic.

The remaining characters like leaf sheath intensity of anthocyanin colouration, flag leaf attitude of the blade (late observation), lemma anthocyanin colouration of keel, anthocyanin colouration of area below the apex, stem length, panicle length of main axis, spikelet colour of tip of lemma, lemma and palea colour, panicle colour of awns, length of awn, panicle attitude of branches, weight of 1000 grain and decorticated grain shape, were found to exhibit large variations in their characters in different genotypes. The presence of purple basal leaf sheath colour, purple auricles, purple leaf ligule, deflexed flag leaf, very strong lemma anthocyanin colouration of apex, light green and purple stigma, deflexed panicle curvature of main axis, well exserted panicles, higher test weight and presence of very low and low amylose content, constitute the distinct features of soft rice genotypes.

It may be concluded from the present investigation that the morphological DUS descriptors can be effectively used for identification and grouping of varieties and varieties satisfying the DUS criteria for these morphological descriptors could be registered under the PPV \& FR Act for obtaining Plant Breeders and Farmers' rights.

\section{References}

Bisne, R and Sarawgi, A.K. 2008. Agromorphological and quality characterization of badshah bhog group from aromatic rice germplasm of Chhattisgarh. Bangladesh Journal of Agriculture Research. 33 (3): 479-492.

Bora, R., Deka, S.D and Sen, P. 2008(a). Identification of rice varieties of Assam, based on grain characters and reaction to certain chemical tests. Seed Research. 36 (1): 51-55.

Bose, L.K and Pradhan, S.K. 2005. Genetic divergence in deep water rice genotypes. Journal of Control, European Agriculture. 6 (4): 635-640.

Chaudhary, R.C and Sahai, V.M. 1993. Collection, evaluation and conservation of combodian rice germplasm. FAO/IBPGR, Plant Genetic Resource Newsletter. 96: 56-53.

Das, S and Ghosh, A. 2010. Characterization of rice germplasm of West Bengal. Oryza. 47 (3): 201-205.

Dhanaraj, K. N., 2001, Morphological and biochemical characterization of seed, seedling and plant in selected genotypes 
of rice (Oryza sativa L.). M.Sc.(Agri) Thesis, University of Agricultural Sciences. Bangalore, Karnataka (India).

Evera, T., 2003, Morphological, biochemical and molecular characterization of rice varieties and hybrids. Ph.D. Thesis. Tamil Nadu Agriculture Univarsity. Coimbatore. Tamil Nadu (India).

Gupta, S. 1990, Physico-chemical characters of some rice cultivars of West Bengal. International Rice Research Newsletter. 15 (1): 12.

Joshi, M.A., Sarao, N.K., Sharma, R.C., Singh, P and Bharaj, T.S. 2007. Varietal characterization of rice [Oryza sativa (L.)] based on Morphological Descriptors. Seed Research. 35 (2): 188-193.

Katsuta, M., Okuna, K., Afzal, M and Anwar, R. 1996. Genetic differentiation of rice germplasm collected in Northern Pakistan. Japan Agricultural Research Quarterly. 30: 61-70.

Mageshwaran, S., 2010, Evaluation of Morphological, chemical and biochemical methods for cultivar identification in rice (Oryza sativa L.) M.Sc. (Agri) Thesis. Tamil Nadu Agriculture University. Coimbatore, Tamilnadu (India).

Mathure, S., Shaikh, A., Renuka, N., Wakte, K., Jawali, N., Thengane, R and Nadaf, A. 2011. Characterisation of aromatic rice (Oryza sativa L.) germplasm and correlation between their agronomic and quality traits. Euphytica. 179: 237-246.

Monika, A., Joshi, A., Navaraj, K., Sharma, R.C., Singh, P and Bharaj, T.S. 2007. Varietal characterization of rice (Oryza sativa L.) based on morphological descriptors. Seed Research. 35 (2): 188193.

Moukoumbi, Y.D., Sie, M., Vodouhe, R., N'dri, B., Toulou, B., Ogunbayo, S.A and Ahanchede, A. 2011. Assessing phenotypic diversity of interspecific rice varieties using agro-morphological characterization. Journal of Plant Breeding and Crop Science 3(5): 74-86.

Nethra, N. 2003. Studies on varietal characterization based on morphological, biochemical and molecular markers in rice. M.Sc. (Agri) Thesis, University of Agricultural Sciences. Bangalore, Karnataka (India).

Reddy, C.A.M. $2005 . \quad$ Varietal characterization by morphological, chemical and electrophoresis of cotton hybrids and their parents. M.Sc. (Agri) Thesis, University of Agricultural Sciences. Dharwad, Karnataka (India).

Rohini Devi, D. 2000. Studies on the characterization of varieties based on morphological and biochemical traits in rice. M.Sc. (Agri) Thesis, University of Agricultural Science. Bangalore, Karnataka (India).

Rosta, K. 1975. Variety determination in rice (Oryza sativa L.). Seed Science and Technology. 3 (1):161-164.

Santhy, V. 1999. Use of biochemical and molecular markers for characterization of parental lines of promising rice (Oryza sativa (L.) hybrids. Ph.D thesis Division of Seed Science and Technology, IARI, New Delhi.

Sarawgi, A.K., Parikh, $M$ and Sharma, B. 2012. Agro-morphological and quality characterization of Dubraj group from aromatic Rice germplasm of Chhattisgarh and Madhya Pradesh. society for plant research. 25 (2): 387394.

Shah, R., Sulemani, M.Z., Balah, M.S and Hassan, G. 1999. Performance of coarse rice genotypes in plains of D.I. Khan, Pakistan. Pakistan Journal of Biological Science. 2: 507-509.

Shaptadvipa, B., Sarma, R.N. 2009. Study on apparent amylose content in context of polymorphism information content along with indices of genetic 
relationship derived through SSR markers in Birain, Bora and Chakuwa groups of traditional glutinous rice of Assam. Asian Journal of Biochemistry. 4 (2): 45-54.

Sharma, D.K., Richharia, A.K and Agarwal, R.K. 2004. Characterisation of ahu rices of Assam for morphological and agronomic traits under transplanted conditions. Oryza. 41 (1\&2): 8-12.

Sharma, K.K., Ahmed, T and Baruah, D.K. 1990. Grain characteristics of some aromatic rice varieties of Assam.
International Rice Research Newsletter. 15 (1): 13.

SubbaRao, L.V., Rana, N.S., Chiranjeevi, M., Chaitanya, U., Sudarshan, I., Suneetha, K., Jyothi, B., Chaudary, R.D. 2013. DUS characterization of rice varieties. Directorate of rice research (DRR). Hyderabad. PP- 524.

Suhasini, K.S. 2006. Characterisation of sesame genotypes through morphological, chemical and RAPD markers. M.Sc.(Agri) Thesis, University Agricultural Sciences. Dharwad, Karnataka (India).

\section{How to cite this article:}

Pragnya, K., K.V. Radha Krishna, L.V. Subba Rao and Suneetha, K. 2018. Studies on Morphological Characterization in Soft Rice (Oryza sativa L.) Genotypes. Int.J.Curr.Microbiol.App.Sci. 7(05): 1348-1374. doi: https://doi.org/10.20546/ijcmas.2018.705.162 